\title{
Research on Computer Remote Network Communication Technology Innovation
}

\author{
Long Yu \\ Dalian Ocean University College of Applied Technology, Dalian, 116023, China
}

Keywords: computer, remote network communication technology, innovation

\begin{abstract}
With the progress of society and the development of the national economy, various modern high-tech technologies have emerged in an endless stream, changing people's past work and lifestyle. Computer remote network communication technology is a communication technology that transmits high-speed and accurate data information. It uses it efficiently and continuously innovates, which can effectively promote the better development of society.
\end{abstract}

\section{Introduction}

With the deepening of research in the field of computer network remote technology, network remote communication technology and network remote control technology have gradually matured, and have been applied in a variety of fields in various fields. From the perspective of technology development, computer network remote communication technology is the basis of computer network remote control technology. Under the circumstances of the Internet, it fully utilizes the technical advantages of the Internet, effectively avoids the security risks existing in the Internet, and realizes network remote communication and control.

\section{The Technical Principle of Remote Network Communication Equipment}

In the application process of computer remote network communication equipment, there are many connection methods, such as a little bit to point, branch point, road duplex and hub type, and the network communication technology is able to play out The inner soul of its own role. The general network communication technology can use the combination of the transmission protocol and the network IO to transmit text, audio and other resources. Generally, the network transmission protocol includes HTTP, TCP, UDP, etc., while the network IO mainly includes NIO, AIO and BIO. After a computer sends out a message, the other computer first responds to the information content, converts the received information into a stream, and then passes it to the receiving end to perform the conversion again, so that the information can be presented on the computer, thereby Achieve remote network communication. There are generally three forms of transmission directions for computer remote network communication, namely simplex communication, duplex communication, and half-duplex communication. The simplex communication mainly refers to the transmission in the direction of information transmission, and the duplex communication refers to the information can be transmitted bidirectionally in the specific transmission process, but the line structure is relatively quite complicated. Half-duplex communication means that information can only be transmitted in two-way information at a certain time or within a certain period of time. At this stage, in the application process of computer remote network communication technology, semi-bidirectional communication is often used for communication, and it has economical and practical universality.

China's computer remote network communication technology has developed rapidly with the improvement of science and technology. At present, computer remote network communication technology relies on operating system, computer technology, communication equipment, etc. to realize fast processing and efficient transmission of signals, and has good data exchange signal capability. Driven by the advantages of the technology machine, it not only realizes efficient transmission and a large amount of information storage process, but also facilitates people's work 
and daily life.

The remote network communication technology can be easily combined with the computer, and the two have better compatibility. The computer auxiliary function is used to realize the signal processing process, and the signal enhancement effect on the communication system is realized, so that the whole communication is realized. The system is more flexible. Moreover, the communication signal of the remote network communication technology in the line is relatively simple, and only a simple circuit design is required to meet the signal quality requirements, which makes the circuit in which it is applied has a simpler requirement and reduces the information transmission. The cost is in line with the level of mass consumption. The combination of remote network communication technology and computer makes the communication system simpler and easier to operate, and has the advantages of simplicity, low failure rate, etc., which can provide good communication quality, can effectively reduce communication cost, improve communication transmission efficiency, and is popular among the public.

From the technical point of view, computer remote network communication is based on the data transmission between computer terminals, the communication content is computer-recognizable network message, and the process of parsing network messages between different terminals under the agreed communication protocol. On the basis of this, the computer remote network control technology increases the process of the computer terminal responding to the message content, and on the basis of the computer remote network control technology, it can form a computer network remote closed-loop control system, which can greatly improve Control accuracy and speed. At present, computer remote network communication and control technology has been promoted in a certain range in various fields of society. In the process of use, its core technologies mainly include network remote wake-up control technology, remote shutdown technology and remote monitoring technology.

\section{Problems and Failures of Computer Remote Communication}

This kind of failure is usually caused by the problem of the device itself. For example, the network port at the connection position between the computer and the switch is recessed, resulting in poor contact and unable to effectively transmit data information. 2 network failure. The main reasons for this failure are: excessive number of people using the network, virus intrusion, and running URL errors. These are also the main problems in remote communication. Too many people using the network will slow down the processing speed of the server, and virus intrusion will lead to system crashes, etc. Running a web address error will prevent the information from being transmitted normally. One of the most important features of computer remote network communication is that information transmission is faster. However, in the big data environment, the explosive development of data will impose a heavy burden on information transmission, which may result in slow information transmission. The application process of the network communication technology is: the user issues the corresponding instruction, and then the system converts it into a symbol, then transmits the symbol, and then converts the signal into an instruction that can be recognized through the switch. If the virtual hard disk that stores user information is attacked, it is very likely to leak information.

The computer remote network communication technology can not only effectively reduce the line cost, improve the communication quality and the transmission efficiency, but also has various characteristics. In the process of computer network communication, computer technology is usually applied in various aspects of the communication field, and the effective connection process between the remote computer network communication technology and the computer integrated circuit can give the whole circuit more choices, and is more convenient. The process of network circuit configuration and control makes the remote network communication diversified.

\section{The Application of Computer Remote Network Communication Technology}

With the rapid development of computer technology, today's computer remote network 
communication technology can realize the operation control and supervision process of the terminal through information sharing in the area, so in the increasingly common life of the national computer network technology and network control equipment. The market demand and application of computer remote network communication technology is very extensive. In many fields, including home digital equipment, agriculture, oil extraction and water treatment technology, computer network communication technology has been applied to realize remote control of equipment. The main function is to remotely turn on and off the equipment. The management process of operating modes such as continuous work. In addition, the use of computer remote network communication technology can quickly realize the sharing analysis and location sharing process of data, facilitating the processing of data information in people's work, and processing the data in the way of computer network data. The data information is more convenient for the content processing process, which improves the work efficiency and reduces the work intensity of the staff, thereby promoting the industrial economic development to a large extent. For example, in the course of our work, the demand for computer-based network communication technology is extremely urgent. It can better improve our office efficiency, enable us to communicate anywhere, anytime, and for each of the work. At the same time, the application of network communication technology also makes wireless communication supervision and management easier and faster, can analyze various data stored in the computer, and realize remote control and operation through far-reaching technology, effectively reducing the work of personnel. The quantity has improved its work efficiency.

\section{Innovation in Computer Remote Network Communication Technology}

When there are problems or failures in the remote network communication technology, it is usually necessary to deal with two aspects: 1 Strengthening the maintenance and maintenance of the physical layer. In the process of maintaining it, the communication port and the network cable must be tested first, and then the corresponding detection technology is combined to effectively maintain various faults in the physical layer, thereby ensuring that the physical layer can be presented. Better function. 2 Implementing detection for the network layer can prove whether the data switch is in a normal state. Then analyze the router and network card to ensure that it can be in a stable working state. For example, when an enterprise applies the TCP/IP protocol, it must build a multi-layer system, and then combine it with the host computer to ensure that the 1-4 interfaces in its S2403 are presented as WLAN A, and the ports 5-17 are presented as WLAN B. The 18-24 interface is presented as a WLAN C, while ensuring that each port can achieve a quality connection with the host. In order to better promote the implementation of network communication technology, comprehensive maintenance is required for the physical layer and the network layer.

As we enter the era of big data, people's daily life and work have shown greater convenience, but there are also some problems in the computer's telecommunications technology, such as communication technology delay or distortion. In order to effectively improve the speed of network communication, the method of expanding the broadband should be used to form a good foundation for the remote network communication of the computer, and then effectively reduce the probability of occurrence of the delay problem and ensure the communication quality. In addition to expanding the broadband, you should also choose a more suitable router and port in combination with the computer, and set a reasonable communication protocol to ensure that the network presents better patency. In addition to ensuring the basic configuration, you should also check the switch and the integrator regularly to ensure that the computer can be in normal operation. When enterprises apply computer network communication technology, if the communication speed is too slow, it will directly lead to the information transmission speed is too low, which is extremely unfavorable for the development of various tasks in the enterprise and future development. In order to increase the speed at which information is transmitted, detailed examinations are required. Enterprises often use multiple computers to work at the same time, which puts higher demands on the network. For example, when a video conference is held, it is necessary to have a larger broadband, which in turn ensures smooth imaging. Therefore, in the era of big data, the use of computer-based remote network communication technology needs to actively combine the actual development needs 
presented by enterprises, gradually improve the network transmission capacity of enterprises, and then better meet the daily operation and development requirements of enterprises. The related technologies of remote network communication are applied to higher quality applications.

In the era of big data, the most important way to innovate related technologies for remote network communication is to construct the information transmission space so that various data information can be transmitted on high-quality channels. At present, the types of lines that are available mainly include cables, overhead lines, and coaxial cable systems, and are specifically set for the specific communication methods. In the process of development, enterprises will generate a very large amount of data information. In order to ensure the security of information, it is necessary to strengthen the application of communication technology, and refer to the actual development of the enterprise to make use of more suitable lines. In order to better transmit data, under the premise of configuring some basic equipment, some more advanced equipment is applied to realize deeper information development.

\section{Conclusion}

All in all, the high-quality application of computer remote network communication technology can effectively promote the development of various industries. Relevant practitioners need to make further research on it, and actively learn from some advanced technologies and concepts from abroad, and the actual situation of the development of China's network communication technology. The combination will inject a steady stream of vitality into the long-term healthy development of our national economy.

\section{References}

[1] Xu Gang, Yang Linhai. Analysis of computer remote network communication technology revolution in the era of big data [J]. Electronic Test, 2017 (18): 61-62+53.

[2] Liu Huihong, Huang Zi, Yang Wenbin. Analysis of Computer Remote Network Communication Technology [J]. Digital Communication World, 2017(07): 46.

[3] Liu Ziwei. Innovation Analysis of Computer Remote Network Communication Technology in Big Data Era [J]. Network Security Technology and Application, 2017(03): 80+82.

[4] Sun Changbo. Research on Transmission Control Technology in Computer Communication [J]. Science \& Technology and Enterprise, 2014(14).

[5] Zhou Jingjing. Discussion on the Application of Computer Communication and Network Remote Control Technology [J]. Information Systems Engineering, 2017(05). 